\title{
Hybrid Concatenated Convolutional Code for Deep Space Mission
}

\author{
Deepak Mishra \\ Onboard Signal Processing \\ Division \\ Space Applications Centre \\ (ISRO)
}

\author{
S.Jit \\ Department of Electronics \\ Engineering \\ Institute of Technology
}

\author{
K S Dasgupta \\ Indian Institute of Space \\ Science and Technology (IIST) \\ Thiruvananthapuram, Kerala
}

\begin{abstract}
In satellite communication deep space mission are the most challenging mission, where system has to work at very low $\mathrm{Eb} / \mathrm{No}$. Concatenated codes are the ideal choice for such deep space mission. ISRO is planning to send unmanned mission for Mars and several deep space missions in future to study and detailed understanding of our own universe. This paper basically reviews the Hybrid concatenated convolutional code structure and selects the suitable candidate for our future deep space mission. The complete simulation using Simulink is done and results are presented in this paper.
\end{abstract}

\section{General Terms}

Iterative decoding, Log-MAP, SIMULINK,Interleaver

\section{Keywords}

Eb/No,PCCCs,HCCCs,SCCCs,SNR,AWGN.

\section{INTRODUCTION}

The usefulness of concatenated codes was first noticed by Forney in [1]. In general, the concatenation of convolutional codes can be classified into three categories,i.e., PCCC, SCCC and hybrid concatenated convolutional codes (HCCC). The constituent convolutional codes (CCs) used in each scheme fall into several classes of systematic, nonsystematic, recursive and non-recursive schemes. Systematic convolutional codes have their inputs appear directly at the output, while non systematic convolutional codes do not have this property. A non-recursive encoder does not have any feedback connection while a recursive encoder does. In general, nonsystematic non-recursive CCs perform almost the same as equivalent systematic recursive CCs since they exhibit the same distance spectrum. In the original turbo code, two identical recursive systematic convolutional (RSC) codes were used. Several other authors have explored the use of nonsystematic recursive $\mathrm{CCs}$ as the constituent codes, e.g., Massey and Costello [2, 3]. In [4, 5],Benedetto et al. and Perez et al. showed that recursive $\mathrm{CCs}$ can produce higher weight output codewords compared to nonrecursive CCs, even when the input information weight is low. This is a major advantage in a PCCC system since low input weight codewords dominate the error events. In addition, PCCC equires long information blocks in order to perform well in the low SNR region. In this case, recursive CCs can provide an additional interleaving gain that is proportional to the length of the interleaver while nonrecursive CCs cannot Therefore, RSCs are preferable in practice as the constituent code for a PCCC or the inner code for an SCCC or HCCC. Detailed treatments of the constituent $\mathrm{CC}$ encoder can be found in Lin and Costello [6] and many excellent references within, e.g., $[4,7]$. In the following sections, we will examine the structure for HCCC scheme. We assume that these systems consist of only two CCs. Extension to multiple CCs is straightforward and have been investigated in a number of references $[8,9]$.

Hybrid concatenated convolutional codes was first proposed by Divsalar et al. [12]. In Divsalar et al. not only the extrinsic information of systematic bits, but also the extrinsic information of parity bits, is exchanged between the component decoders. Therefore, the performances of HCCC have been shown to be superior, in some cases, to a general turbo code.

In 2003, Benedetto et al. [13] compared the structure of three covolutional concatenated codes. They presented an analytical struture of three codes.

In 2008, Koller et al. [14] investigated hybrid concatenated coding structures consisting of an outer multiple parallel concatenated codes (MPCCs) with very simple memory-1 component encoders serially concatenated with an inner accumulator. They have shown that such structures exhibit linear distance growth with block length and they have better thresholds than multiple serially concatenated codes (MSCCs). The results indicate a fundamental tradeoff between minimum distance growth and convergence threshold in turbo-like codes.

Gergis [15] presented a number of powerful recent classes of serial and parallel concatenated trellis codes that have been analyzed and compared with a proposed third choice called hybrid concatenated trellis code HCTC. These comparisons show the superiority of HCTC over the classical SCCC and PCCC schemes. They have also demonstrated that there is a significant increase in the performance and decrease in the bit error rate and probability of errors of HCTC with increasing: the interleaver size $N$, the code constraint length, and the number of decoder iterations. 
Oluwafemi et al. [16] proposed two hybrid concatenated super-orthogonal space-time trellis code (SOSTTC) applying iterative decoding for flat fading channels. The encoding operation is based on the concatenation of convolutional codes, interleaving and super-orthogonal space-time trellis codes. The first concatenated scheme consists of a serial concatenation of a parallel concatenated convolutional code with a SOSTTC while the second consist of parallel concatenation of two serially concatenated convolutional and SOSTTC codes. They have shown that proposed topologies perform better than existing concatenated schemes with constituent code of convoluntional and space time codesIn 2012, Mansour et al. [17] proposed a special construction of concatenated convolutional coding scheme called parallel-serial concatenated convolutional code (P-SCCC). They have evaluated the upper bound to the bit error probability of the proposed code. The error performance of this proposed code scheme is better than that of both classical serial and parallel concatenated convolutional codes.

Cheng et al. [Cheng et al.(2008)] improved the performance of hybrid convolutional concatanated codes by introducing $a$ modified Log-MAP algorithm. The new coding scheme achieve about $1.0 \mathrm{~dB}$ additional coding gain, compared to the general turbo coding scheme at a BER $=10^{-6}$, with a frame length of 8192-bit. The system complexity and decoding latency of the new scheme is lower than the HCCC proposed by Divsalar et al. However at lower Eb/No the performance of proposed modified Log-MAP is inferior to Divsalar et al. proposed algorthim.

\section{DRAWBACK OF SERIAL AND PARALLEL CONCATENATED CODES}

All Performance of serial and parallel concatenated convolutional schemes with iterative decoding techniques for different interleaver designs were investigated in [10, 11]. Figure 1[10] shows initial results obtained for the parallel and serial concatenated schemes, respectively, based on inner and outer convolutional codes, and 8 iterations.

For the parallel concatenated scheme illustrated in Figure 1, both the inner and outer codes are identical rate 2/3 16 state RSC codes. For the serial concatenated scheme, the outer code is the same RSC code as used in the parallel scheme, while the inner code is a rate $3 / 416$ state RSC code. Serial and parallel schemes having the same delay $(60,600,6000)$ are compared. It is characteristic for PCCC schemes to perform better than SCCC schemes at low SNRs. However, increasing the SNR, SCCC schemes outperform PCCC

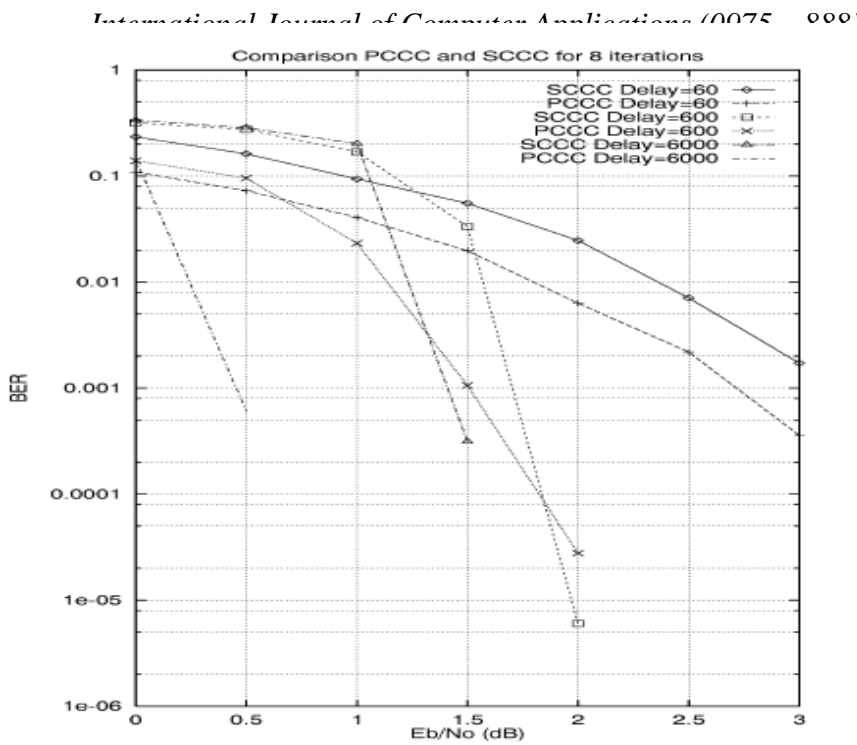

schemes. The cross-over point depends on the interleaver size and interleaver design.

So the final conclusion is that PCCCs perform exceptionally well at low signal-to-noise ratios (SNRs) but develop rather high error floors at high SNRs [3]. On the other hand, SCCCs can achieve extremely low bit error rates at high SNRs, although this comes at the cost of worse performance (relative to PCCCs) at very low SNRs [4].

\section{Figure 1: PCCC and SCCC Comparison}

\section{HYBRID CONCATENATED CODES}

A hybrid concatenated code with two interleavers is the parallel concatenation of an encoder, which accepts the permuted version of the information sequence as its input, with a serially concatenated code, which accepts the unpermitted information sequence. Hybrid concatenated code is first proposed by Divsalar-Pollara[12],However performance of hybrid concatenated code is furthur improve by using modified Log -MAP algorthim proposed by YaCheng Lu, Erl-Huei Lu [18].In modified Log-MAP algorthim both the extrinsic information of systematic bits and parity bits can be retrieved during iterative decoding. However at lower SNR condition performance of Divsalar proposed HCCC structure is performed better compared to modified Log-Map algorthim. In this paper we will discuss the performance of Divsalar proposed HCCC struture As for the SCCC structures, the interleaver gain for the HCCC depends on the free distance of the outer code in the serial concatenation part of the HCCC. The multiplication factor is $\mathrm{N}^{-}{ }^{\text {do }}$ for the BER bound, where do is the free distance of the outer code. Therefore the HCCC structure is a further improvement on the SCCC structure.

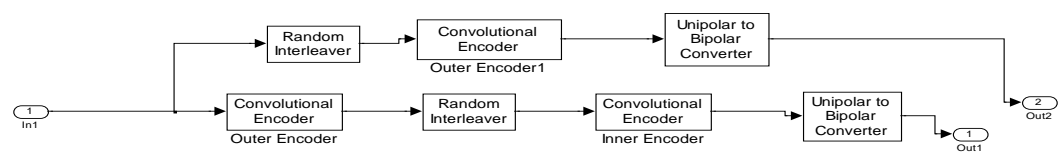

Figure 2.a. SIMULINK Model of Hybrid Concatenated Encoder 


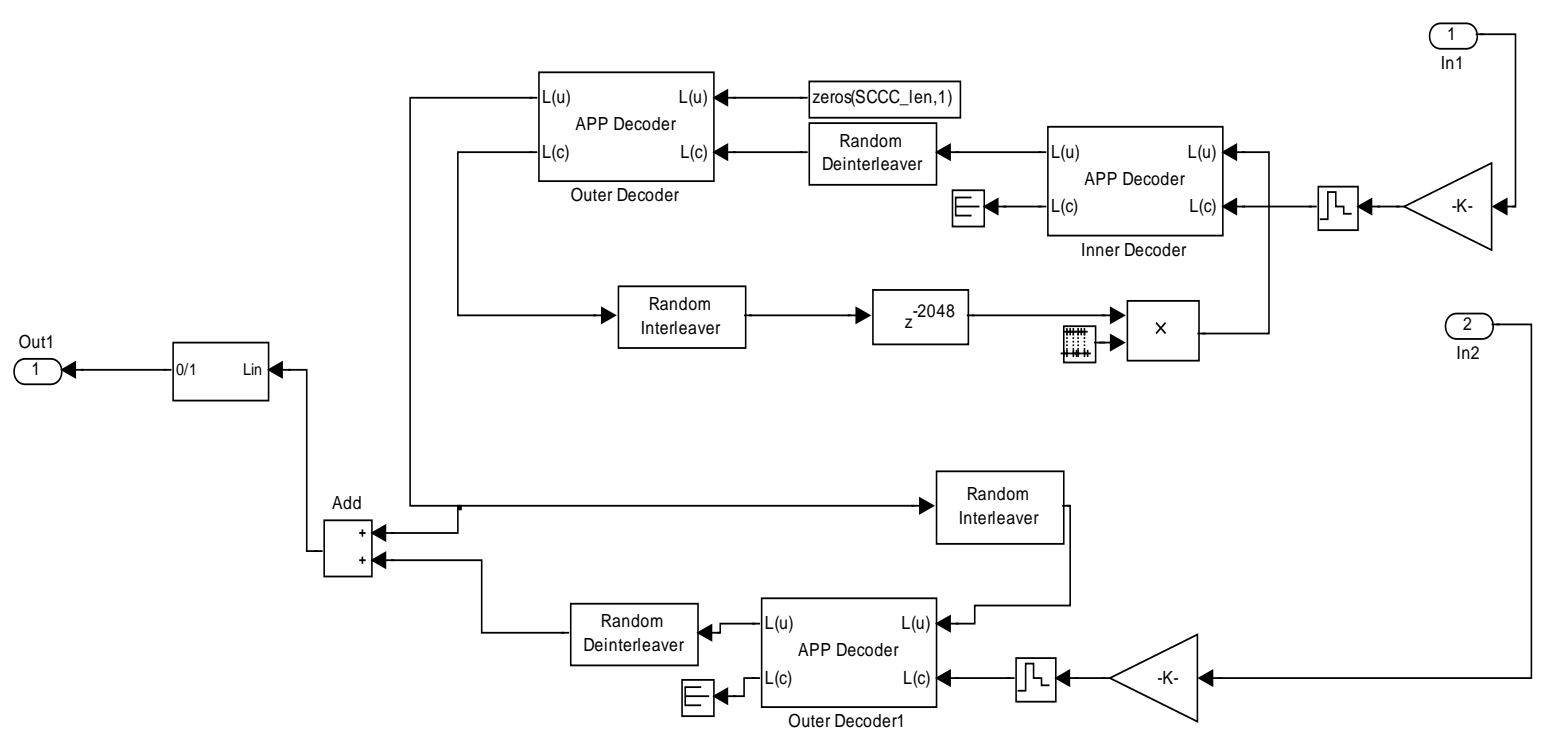

Figure 2.b. SIMULINK Model of Hybrid Concatenated Decoder

As per proposed structure by Divsalar-Pollara figure 2.a and 2.b shows the SIMULINK model of hybrid concatenated encoder and decoder

\section{SIMULATION RESULT}

Figure 3 shows the comparative performance of all three types of concatenated code structure.The simulation parameter kept identical in all three cases.
The code rate for all three cases is $1 / 2$ and total decoding iteration is set to 6 . The frame length is set to 1784 Bits .In all three cases random interleaver and deinterleaver is used. The comparative performance of all three type structure is shown in Figure 4.

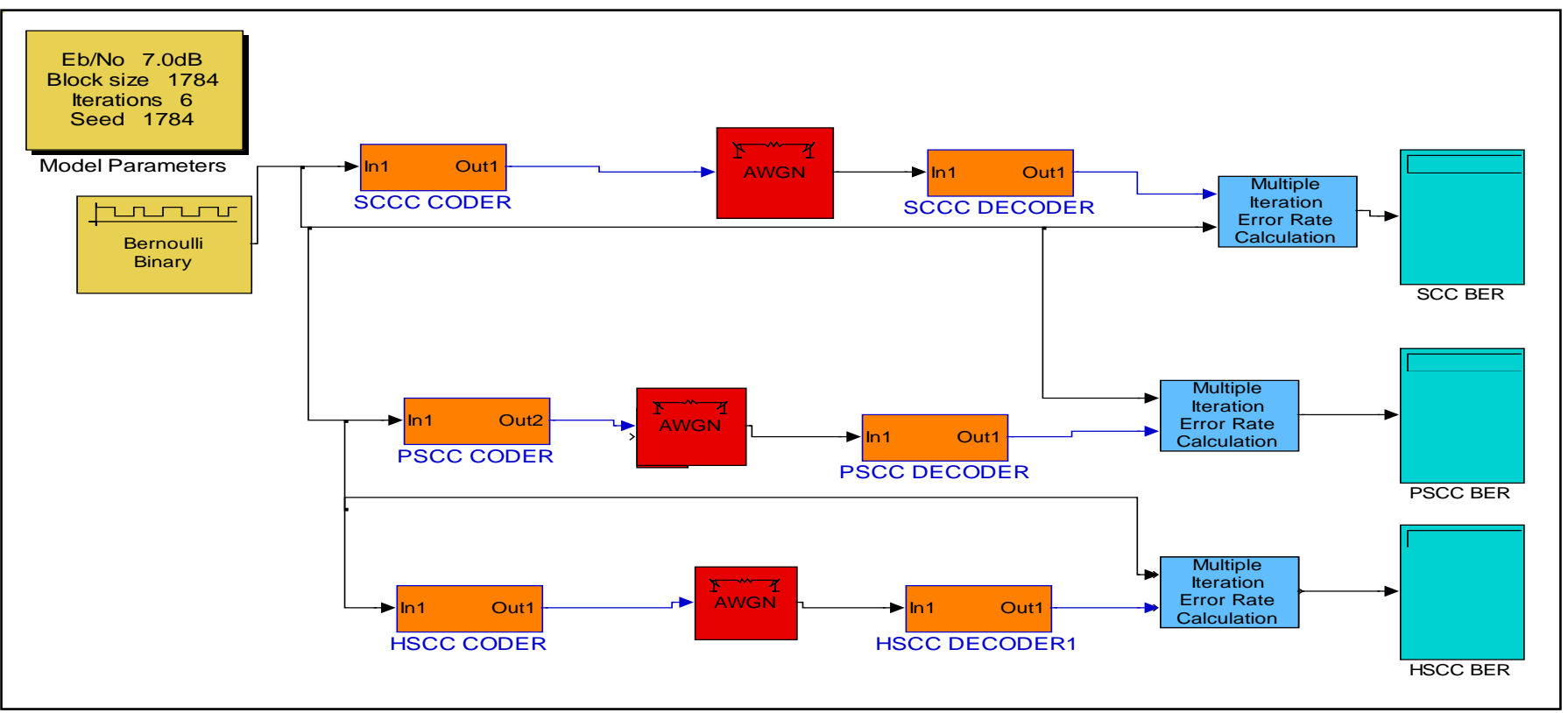

Figure 3. Comparitive Performance Analysis of PSCC, SCCC and HSCC Codec. 


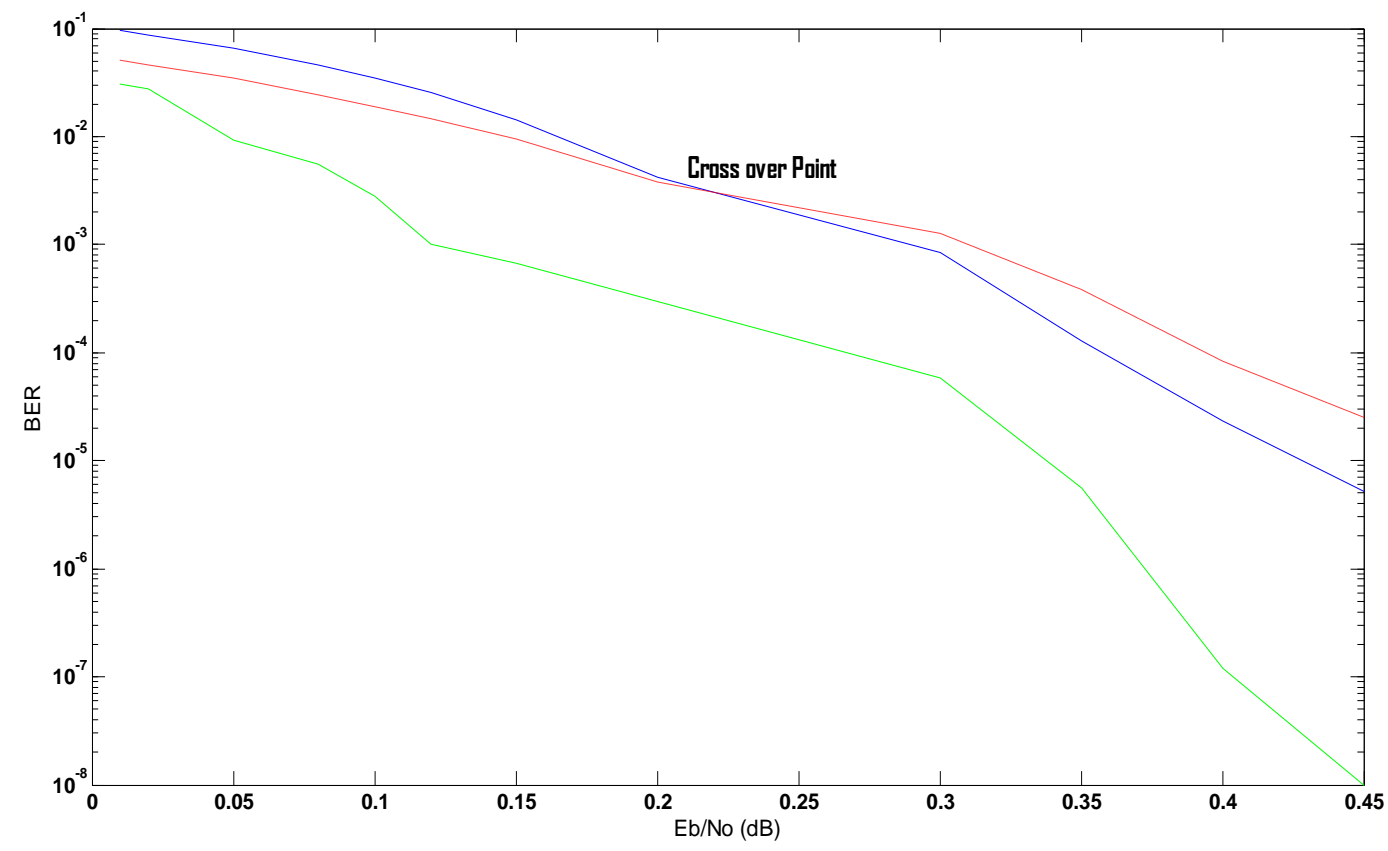

Figure 4 .Comparitive Performance of Concatenated Codes

The comparative performance in terms of coding gain with QPSK modulation scheme is mention in Table 1. It can be easily seen that the serial concatenated performance is superior compare to parallel concatenated code at higher $\mathrm{Eb} / \mathrm{No}$, while at lower Eb/No performance of parallel concatenated code is better. It may be noted that cross over point of SCCCs and PCCCs is depend upon frame length, type of interleaver and number of iteration. The performance of hybrid concatenated code is superior at all $\mathrm{Eb} / \mathrm{No}$. Hence hybrid concatenated code is the suitable candidate for deep space mission. The key point in the performance of all three codes is iterative decoding. In order to verify how number of iteration will improve the performance Figure 5 will shows the performance of hybrid concatenated code with respect to number of iteration.
TABLE 1. Comparative Performance of Coding Gain

\begin{tabular}{|l|l|l|l|l|}
\hline Type & $\begin{array}{l}\text { Coding } \\
\text { Gain } \\
\text { at 10 } \\
\text { BER }\end{array}$ & $\begin{array}{l}\text { Coding } \\
\text { Gain } \\
\text { at 10 } \\
\text { BER }\end{array}$ & $\begin{array}{l}\text { Initial } \\
\text { decoding } \\
\text { Delay } \\
\text { (ms) }\end{array}$ & Performance \\
\hline SCCC & $7.6(\mathrm{~dB})$ & $9.2(\mathrm{~dB})$ & 5.00 & $\begin{array}{l}\text { Performance } \\
\text { better at } \\
\text { Higher Eb/No }\end{array}$ \\
\hline PCCC & $8.0(\mathrm{~dB})$ & $8.4(\mathrm{~dB})$ & 3.9 & $\begin{array}{l}\text { Performance } \\
\text { better at } \\
\text { Higher Eb/No }\end{array}$ \\
\hline HCCC & $9.0(\mathrm{~dB})$ & $9.8(\mathrm{~dB})$ & 6.50 & $\begin{array}{l}\text { Performance } \\
\text { better at } \\
\text { Higher as well } \\
\text { as Lower } \\
\text { Eb/No }\end{array}$ \\
\hline
\end{tabular}

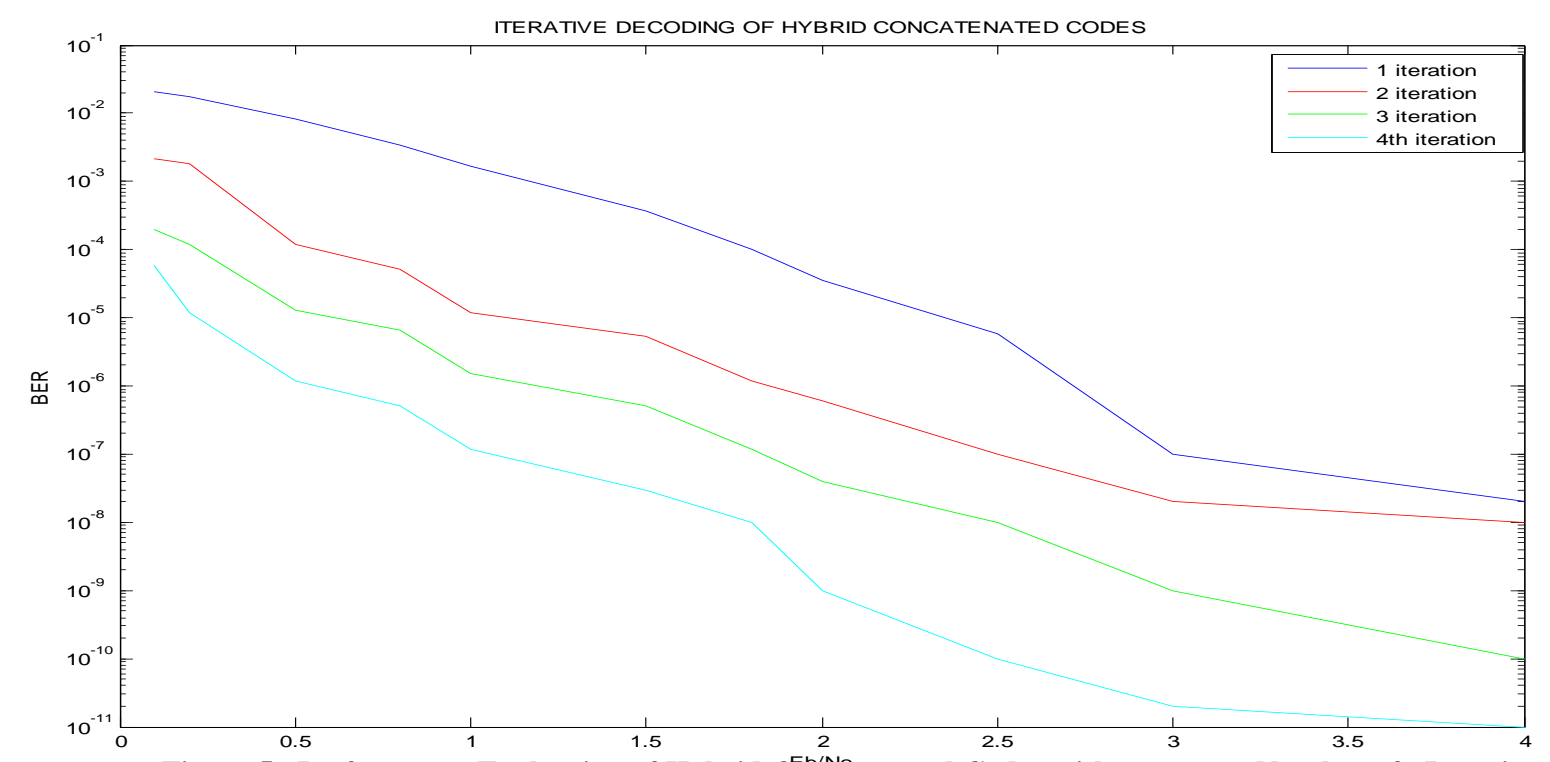

Figure 5. Performance Evaluation of Hybrid Conchatenated Codes with respect to Number of Iteration 
It can be easily seen that as the number iteration increases the performance of HCCC concatenated code increases drastically. However as the number of iteration increases the decoding delay and complexity increase also increase proportionally.

Now we know that selection of interleaver of HSCC codec with respect to different interleavers. It can be easily seen that performance of random interleaved HSCC codec is superior compare to other interleaved HSCC codec. Hence random interleaver is the suitable choice for HSCC codec.
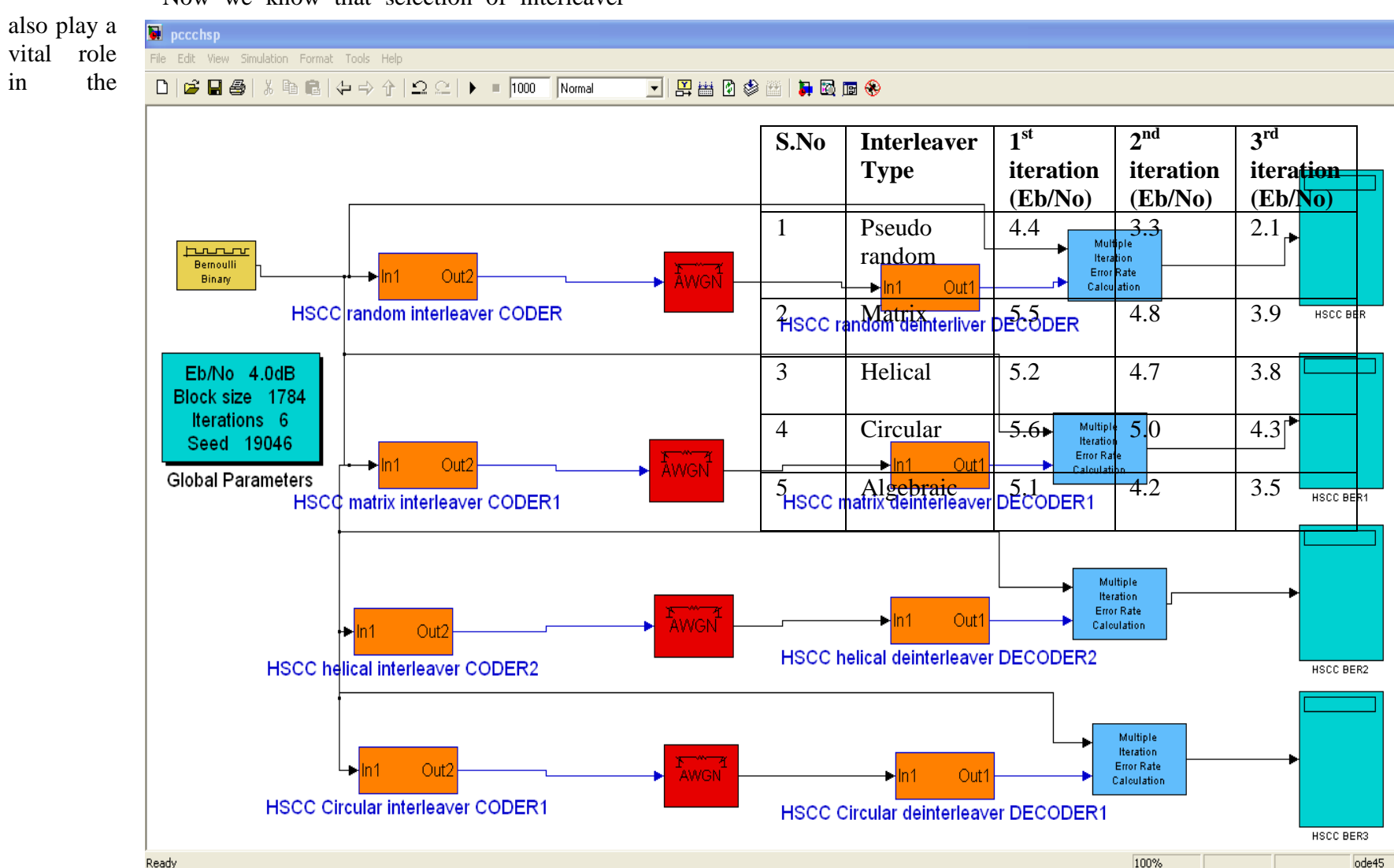

performance of concatenated codes. Figure 6 show comparative performance analysis of HCCC code using random, matrix, helical and circular interleaver. Table 2 shows the error free performance analysis
TABLE 2: Error Free Performance of HSCC codec with respect to different Interleavers Packet size : 1784 Bits, BER $=10^{-6}$

Figure 6. Comparative Performance Analysis of Hybrid Concatenated Codes with respect to different Interleavers

\section{CONCLUSION}

A detailed simulation result are presented for all three concatenated code structure for ISRO deep space mission . Simulation result shows that for identical code rate, the performance of Hybrid concatenated code is superior compare to serial and Parallel concatenated code structure. However complexity of Hybrid concatenated decoder is higher compare to other concatenated code structure. Simulation result also shows that random interleaver is the ideal choice for hybrid concatenated code structure. Hence random interleaved Hybrid concatenated code is suitable candidate for ISRO deep space mission.

\section{REFERENCES}

[1] Forney, G. D., Jr., Concatenated Codes (Cambridge, MA: MIT Press, 1966).

[2] P. C. Massey and D. J. Costello, Jr., "Turbo codes with recursive nonsystematic quick-look-in constituent codes," IEEE Int. Symp. Inform. Theory, Washington, USA, pp. 141-145, June 2001.

[3] A. Banerjee, F. Vatta, B. Scanavino, and D. J. Costello, Jr., "Nonsystematic turbo codes," IEEE Trans. Commun., vol. 53, pp. 1841-1849, Nov. 2005.

[4] S. Benedetto, D. Divsalar, G. Montorosi, and F. Pollara, "Serial concatenation of interleaved codes: Performance analysis, design and iterative decoding,"IEEE Trans. Inform. Theory, vol. 44, pp. 909-926, May 1998.

[5] L. C. Perez, J. Seghers, and D. J. Costello, Jr., "A distance spectrum interpretation of turbo codes," IEEE Trans. Inform. Theory, vol. 42, pp. 1698-1709, Nov. 1996.

[6] S. Lin and D. J. Costello, Jr.,"Error control coding," Pearson Prentice Hall, New Jersey, 2004.

[7] S. Benedetto, R. Garello, G. Montorsi, C. Berrou, C. Douillard, D. Giancristofaro,A. Ginesi,L. Giugno and M. Luise, "MHOMS: High speed ACM modem for satellite applications," IEEE Trans. Wireless Commun., vol. 12,pp. 66-77, Apr. 2005. 
[8] D. Divsalar and F. Pollara, "Multiple turbo codes," IEEE Military Commun.Conf., San Diego, USA, pp. 279-285, Nov. 1995.

[9] S. Huettinger and J. Huber, "Design of multiple-turbocodes with transfer characteristics of component codes," Conf. on Inform. Sciences and Systems,Princeton, USA, Mar. 2002

[10] S. Benedetto, G. Montorsi, D. Divsalar and F. Pollara, "Serial concatenation of interleaved codes: Performance Analysis, Design, and Iterative Decoding", JPL TDA Progress Report 42-126, August 15, 1996.

[11] Gray "Serially concatenated trellis coded modulation", PhD Dissertation, Univ. of South Australia, submitted, Aug. 1998.

[12] D. Divsalar and F. Pollara, "Hybrid concatenated codes and iterative decoding," JPL TDA Progress Rep. 42-130, pp.1-23, Aug. 151997.

[13] Benedetto, S.; Montorsi, G.; Divsalar, D. "Concatenated convolutional codes with interleavers," IEEE Communications Magazine,Volume: 41 , Issue: 8 , Aug. 2003 Pages:102 - 109.

[14] Koller .C, A. Graell i Amat, J. Kliewer, F. Vatta, D. J. Costello, Jr., "Hybrid Concatenated Codes with Asymptotically Good Distance Growth", in Proc. IEEE
International Symposium on Turbo Codes \& Related Topics, pp. 19-24, September 2008.

[15] Gergis Labib Francis," Performance of Hybrid Concatenated Trellis Codes CPFSK with Iterative Decoding over Fading Channels" International Journal of Distributed and Parallel systems. ISSN :0976 - 9757 [Online] ; 2229 - 3957 [Print].

[16] Oluwafemi .Ilesanmi B. and Stanley H. Mneney, "Hybrid Concatenated Super-Orthogonal Space-Time Trellis Codes Applying Iterative Decoding," IEEE AFRICON 2011 Livingstone, Zambia $13^{\text {th }}-15^{\text {th }}$ September 2011.

[17] Mansour Hala M. A., Labib Francis Gergis, Mostafa A. R. Eltokhy, Hoda Z. Said, "Performance Analysis for Concatenated Coding schemes with Efficient Modulation Techniques" International Journal of Soft Computing and Engineering (IJSCE) ISSN: 2231-2307, Volume-2, Issue-1, March 12.

[18] Ya-Cheng Lu,Erl-Huei Lu, “ Enhancement of Concatenated Codes Using A modified LogMAPAlgorithm", Proceeding ICICC'08 Proceeding of the 2008 3rd International Conference on Innovative Computing Information and Control 